\title{
Las motivaciones de los turistas LGBT en la elección de la ciudad de Barcelona
}

\author{
Josep Maria Prat Forga \\ Universitat Autònoma de Barcelona. Departament de Geografia \\ josepmaria.prat@uab.cat
}

Recepción: marzo de 2015

Aceptación: junio de 2015

\section{Resumen}

El turismo LGBT se está consolidando como una importante actividad dentro del sector turístico. La ciudad de Barcelona no es ajena a esta tendencia. Sin embargo, la satisfacción obtenida con esta experiencia depende de diferentes variables según la motivación principal para realizar este viaje. En este artículo se analizan estos aspectos comparando los resultados de dos encuestas. La primera, a una muestra de los asistentes al Festival Circuit, y la segunda, a un grupo de turistas LGBT ajenos a este evento. De esta manera se ha confirmado que hay significativas diferencias entre la valoración dada por estos dos grupos de turistas a las diferentes variables que influyen en su viaje. El aspecto afectivo es el más valorado por los que realizan el viaje sin el reclamo de un gran evento pensado para este colectivo, aunque el componente lúdico tiene una gran influencia entre los asistentes a un macroevento pensado para el colectivo LGBT.

Palabras clave: turismo LGBT; satisfacción visitas; festivales musicales; motivaciones; Barcelona.

Resum. Les motivacions dels turistes LGBT en l'elecció de la ciutat de Barcelona

El turisme LGBT s'està consolidant com una important activitat dins del sector turístic. La ciutat de Barcelona no és aliena a aquesta tendència. No obstant això, la satisfacció obtinguda amb aquesta experiència depèn de diferents variables segons la motivació principal per realitzar aquest viatge. En aquest article s'analitzen aquests aspectes comparant els resultats de dues enquestes. La primera, a una mostra dels assistents al Festival Circuit, i la segona, a un grup de turistes LGBT aliens a aquest esdeveniment. D'aquesta manera s'ha confirmat que hi ha diferències significatives entre la valoració donada per aquests dos grups de turistes a les diferents variables que influeixen en el seu viatge. L'aspecte afectiu és el més valorat pels que realitzen el viatge sense el reclam d'un gran esdeveniment pensat per a aquest col-lectiu, encara que el component recreatiu té una gran influència entre els que assisteixen a un gran esdeveniment preparat per al col-lectiu LGBT.

Paraules clau: turisme LGBT; satisfacció visites; festivals musicals; motivacions; Barcelona. 
Résumé. Les motivations des touristes LGBT dans le choix de la ville de Barcelone

Le tourisme LGBT s'est consolidé en tant qu'activité importante à l'intérieur du secteur touristique. La ville de Barcelone n'est pas étrangère à cette tendance. Cependant, la satisfaction obtenue avec cette expérience dépend de différentes variables selon la motivation principale pour réaliser ce voyage. Dans cet article, ces aspects sont analysés en comparant les résultats de deux enquêtes. La première, réalisée auprès d'un échantillon des assistants au Festival Circuit et, la deuxième, auprès d'un groupe de touristes LGBT étrangers à cet évènement. De cette façon, nous avons une confirmation du fait qu'il existe des différences significatives entre l'évaluation donnée par ces deux groupes de touristes aux différentes variables qui déterminent leur voyage, l'aspect affectif étant plus importants pour ceux qui réalisent le voyage sans la réclame d'un grand évènement pensé pour ce collectif, bien que la composante récréative ait une influence importante sur les assistants à un macro évènement pensé pour le collectif LGBT.

Mots-clés: tourisme LGBT; satisfaction visites; festivals musicaux; motivations; Barcelone.

\section{Abstract. Motivations of LGBT tourists in choosing the city of Barcelona}

LGBT tourism has emerged as an important activity within the tourism sector. Although this trend is not new to the city of Barcelona, the satisfaction of LGBT tourists depends on different variables related to the principal motivation for travel. This article analyzes such aspects by comparing the results of two surveys: the first of a sample of attendees at the Barcelona Circuit Festival and the second of a group of LGBT tourists who have not attended this event. Significant differences are found between how these two groups of tourists evaluate the variables influencing the trip. The affective aspect is most valued by those who travel without the attraction of a large-scale event targeted at this group, although leisure has an important influence on attendees at a macro event organized specifically for the LGBT community.

Keywords: LGBT tourism; satisfaction with visit; musical festivals; motivations; Barcelona.

\section{Sumario}
1. Introducción
4. Resultados
2. Barcelona y el turismo LGBT
5. Conclusiones
3. Metodología
Referencias bibliográficas

\section{Introducción}

El turismo se ha convertido en una poderosa arma de desarrollo económico para muchos países, lo que, junto con los cambios en los hábitos de unos turistas cada vez más experimentados y que demandan unos productos más personalizados, ha provocado profundos cambios en el sector, con diversos tipos de turistas (Troitiño et al., 2003; Prat y Cànoves, 2012). Las grandes ciudades han entendido este fenómeno y su apuesta por satisfacer esta nueva demanda se ha ido consolidando en los últimos años (Fernández Salinas, 2007; 
González y Morales, 2009; Barrado, 2010; Smith, 2012). Ahora bien, para que estos nuevos productos sean competitivos en el entorno actual, globalizado, es imprescindible conocer lo más detalladamente posible las motivaciones de estos turistas para escoger un destino turístico (Martínez Quintana, 2006; Salazar, 2012).

El turismo LGBT no es ajeno a este fenómeno. Las siglas LGBT designan colectivamente a lesbianas, gays, bisexuales y transexuales, con el objetivo de englobarlos en las diversas culturas basadas en la sexualidad y la identidad de género (Shankle, 2006). Se trata de un tipo de turismo con unas motivaciones y necesidades concretas y unas variables emocionales específicas que influyen decisivamente en la satisfacción con la experiencia realizada en un determinado destino turístico (Russell, 2001; Jensen-Campbell, 2004; Waitt y Markwell, 2006).

El espacio desempeña un papel fundamental en la creación de la identidad sexual (Forest, 1995; Cooper. 2010) y aunque algunos autores defienden la globalización del turismo homosexual y la "desestigmatización» de ciertos destinos turísticos (Oswin, 2006), tradicionalmente el turista LGBT ha elegido dos grandes tipos de destinos: los de sol y playa y las grandes ciudades. Ambos presentan una amplia oferta de atracciones y recursos dirigidos al público LGBT. De este modo, el «lugar», que es una articulación particular del proceso social, entendiéndose como «la ubicación de conjuntos particulares de relaciones sociales intersectadas» (Massey et al., 1995), se transforma en un ente dinámico, con una evolución permanente tanto de actividades como de relaciones sociales. Por ello, los lugares son constructores activos de los procesos sociales y dan significado a sus actividades (Massey, 1994; Cresswell, 1996).

Ante esta circunstancia, y teniendo en cuenta que el turismo LGBT se ha consolidado en la ciudad de Barcelona (España) en estos últimos tiempos (Moner, Royo y Ruiz, 2006), el objetivo del presente trabajo es analizar los aspectos que valoran más estos turistas a lo largo de su estancia en este destino y determinar cuáles son las diferencias motivacionales entre los turistas LGBT que realizan el viaje bajo el reclamo de algún macroevento de gran atracción internacional diseñado para este colectivo y los que no lo hacen por este motivo. De esta manera, se ayudará a los organismos públicos y a las empresas privadas relacionadas con el desarrollo de este turismo a conocer y promocionar los aspectos que valoran más los turistas LGBT para visitar la capital catalana.

Para ello se plantean tres hipótesis. La primera es que hay significativas diferencias entre las motivaciones de los turistas LGBT que realizan el viaje bajo la atracción de algún macroevento y los que no, de modo que la valoración dada por ambos grupos a las diferentes variables que influyen en su viaje es distinta (H1). La segunda es que el aspecto más valorado por los turistas LGBT que realizan el viaje sin el reclamo de un gran evento es el afectivo ${ }^{1}(\mathrm{H} 2)$. La ter-

1. El sexo tiene una importancia fundamental porque los seres humanos tienen la capacidad de vincularse con los demás, y la sexualidad facilita esta vinculación, resuelve las necesidades de intimidad, de afecto, de cariño, de ternura, de contacto y de placer (Pino, 2010). 
cera es que el componente lúdico tiene una gran importancia para los turistas LGBT que acuden a un macroevento diseñado para este colectivo $(\mathrm{H} 3)$.

Para ello, después de revisar la situación actual del desarrollo del turismo LGBT en la ciudad de Barcelona, se comparan y analizan los resultados de dos encuestas realizadas personalmente a estos dos grupos de turistas LGBT durante su estancia en Barcelona. Una, realizada en 2012, a una muestra de los asistentes al Festival Circuit de aquel año (se trata de un festival internacional de actividades de ocio pensadas para los colectivos LGBT que se celebra anualmente en Barcelona desde 2008) y cuyos resultados fueron publicados en la revista Cuadernos de Turismo (Prat, 2014). Otra, realizada en el año 2014 a otra muestra de turistas LGBT que se encontraban en aquel momento en la ciudad pero sin ningún gran evento que les hubiese servido como reclamo para hacer el viaje.

\section{Barcelona y el turismo LGBT}

La ciudad de Barcelona ha apostado decididamente por el turismo como uno de sus principales motores de desarrollo económico. El turismo LGBT no ha sido ajeno a esta tendencia, aunque desde 1970 ya era lugar de atracción del movimiento clandestino de lesbianas, gays, transexuales y bisexuales (Moner et al., 2006; Prat, 2014). Sin embargo, fue en los años ochenta del pasado siglo cuando empezó a ser considerada un destacado destino internacional para los turistas LGBT (Moner et al., 2006; Turisme de Catalunya, 2013).

Los factores por los que la ciudad de Barcelona se ha asentado como un destino destacado para estos turistas son la existencia de una adecuada seguridad personal, el reconocimiento legal desde el año 2005 de las parejas del mismo sexo, la tolerancia, respeto y mente abierta de sus residentes para con estos colectivos, la presencia de un importante patrimonio cultural urbano, el clima templado, el sol y las playas, una reputada gastronomía, su amplia oferta hotelera, sus comercios y las diferentes actividades que periódicamente allí se celebran (deportivas, de ocio y recreación, congresos, etc.) (Hosteltur, 2007; Prat, 2014).

La relación entre sexo y turismo ${ }^{2}$ ha adquirido últimamente gran importancia en la investigación académica y muchos autores se han concentrado en los aspectos más económicos de estas relaciones (entre otros, Knopp, 1998; Buhalis, 2000; Pritchard y Morgan, 2000; Jeffreys, 2003; Beddoe, 2003; Waitt y Markwell, 2006; Monterrubio, 2008), en los aspectos motivacionales (como Waitt y Gorman-Murray, 2008; Prat, 2010, 2014; Prat y Cànoves, 2012) y también en el desarrollo de productos turísticos complementarios (McKercher y Bauer, 2003; Norrild, 2007). Otros, como Forest (1995), Visser (2002), Fernández Salinas (2007), Hughes (2006), Wall y Mathieson (2006) y Monterrubio (2008), han analizado los efectos reales y potenciales de este turismo en las comunidades receptoras.

2. La relación entre sexo y turismo es un fenómeno polifacético relacionado con la experiencia turística de mucha gente y que, a veces, puede realizarse de manera consentida y no comercializada (Tubella y Vilaseca, 2005). 
El turismo LGBT se caracteriza por una creciente demanda de nuevos servicios teniendo en cuenta que se trata de una modalidad en la que confluyen varios colectivos con diferentes motivaciones, aunque todos confluyen en una concentración espacial discreta (bares y clubs especializados, hoteles, tiendas y espacios públicos) donde se permiten las interrelaciones personales (Edensor, 2001; Hughes, 2002; Gibson, 2012; Prat, 2014).

En general, sus practicantes suelen ser personas solteras o separadas sin hijos bajo custodia directa, con un nivel social, económico y cultural medio y medioalto, una gran capacidad para viajar en cualquier época del año y que pueden permitirse realizar estos viajes gastando más que la media de los turistas (Prat, 2014), ya que su poder adquisitivo se estima en 1.356 millones de euros a escala mundial (Matinée Group, 2012). Se trata de un turismo que mueve más del $10 \%$ del volumen mundial de turistas, con más de 700 millones de personas, que gastan al año más de 185.000 millones de dólares en esta actividad, lo que representa el 15,96\% del gasto total del sector. Además, su ritmo de crecimiento es del 10,3\% anual, frente al 3,8\% del turismo en general (OMT, 2014).

Los turistas LGBT suelen escoger destinos urbanos donde sean bien acogidos y puedan sentirse cómodos en compañía de otras personas del mismo género. Disponen de la oportunidad de vivir una sexualidad abierta y sin prejuicios, realizar un consumo cultural y recreativo satisfactorio, poder relajarse y tener la máxima libertad posible (Pritchard et al., 2000; Hughes, 2002; JensenCampbell, 2004; White y White, 2008). Por ello, algunas grandes ciudades, como Barcelona, han apostado por ofrecer sin restricciones nuevos productos turísticos orientados específicamente a satisfacer las demandas de este colectivo. Entre ellos destacan los festivales de música (Pritchard et al., 2000).

Una prueba de la importancia que poco a poco está adquiriendo este turismo se refleja en la Feria Internacional de Turismo (Fitur). Una feria que se celebra anualmente en Madrid para que las empresas que se dedican al sector del turismo puedan intercambiar ideas o lazos comerciales. Así, debido al desarrollo que ha experimentado el turismo LGBT, desde hace cinco años Fitur también celebra una feria temática (Fitur LGBT) pensada específicamente para este colectivo.

Por otra parte, en general, los residentes en los destinos que reciben este turismo suelen aceptarlo positivamente, ya que genera un importante impacto económico (hoteles, transportes, restaurantes, discotecas, comercios, etc.) (Waitt et al., 2008). Sin embargo, también pueden aparecer impactos negativos, como un aumento de tensiones en la ocupación de los espacios públicos (como las playas o las discotecas), ya que el concepto de heteronormatividad ${ }^{3}$ distingue los espacios según su mayor o menor grado de conformidad con el modelo sexual dominante (Raibaud, 2007; Prat, 2010, 2014). A pesar de ello,

3. El concepto hace referencia «al conjunto de las relaciones de poder por medio del cual la sexualidad se normaliza y se reglamenta en nuestra cultura y las relaciones heterosexuales idealizadas se institucionalizan y se equiparan con lo que significa ser humano». Es decir, la heteronormatividad es el régimen social y cultural que impone que la heterosexualidad sea la única sexualidad «normal», natural y aceptada (Jeyasingham, 2011). 
los espacios urbanos ocupados por estos turistas han sido aceptados de forma gradual por la comunidad local, bajo el apoyo de los organismos públicos. De esta forma, las ciudades cumplen con su función de atracción turístico-cultural y con un determinado modelo urbano (Benach y Tello, 2004).

En su apuesta por el turismo, Barcelona ha promocionado una imagen de ciudad atractiva y multicultural al multiplicar las zona verdes, mejorar la limpieza de las calles, redistribuir el flujo automovilístico, potenciar el transporte público, ampliar el aeropuerto y el puerto, promocionar el uso de la bicicleta, facilitar la construcción de hoteles, aprobar una apertura horaria de los comercios y la instalación de tiendas, restaurantes y cafeterías en las zonas más emblemáticas de la ciudad y subvencionar la restauración de las fachadas de los viejos edificios (Borja, 2010). Además, uno de los atractivos principales de Barcelona no solo es su Barrio Gótico y sus edificios modernistas sino también su cultura moderna. Muchas personas acuden a esta ciudad en busca de nuevas expresiones artísticas, nuevos sonidos musicales y nuevas maneras de relacionarse (Tomsen y Markwell, 2007).

Este auge turístico también ha repercutido en el colectivo LGBT, que ha convertido la ciudad en un destino turístico gay-friendly, con 250 recursos pensados especialmente para uso y disfrute de este colectivo (hoteles, bares y cafeterías, comercios, discotecas), y ha dado trabajo directo a más de dos mil personas. De este modo, la capital catalana recibió en el 2013 más de 500.000 turistas LGBT que generaron 480 millones de euros (Turisme de Catalunya, 2013). Así pues, los turistas LGBT se han consolidado ya como uno de los segmentos más importantes del turismo que recibe Barcelona, y su importancia cuantitativa es comparable a la de quienes llegan en crucero o lo hacen atraídos por el modernismo.

Desde las instituciones públicas catalanas también se ha favorecido este desarrollo, como es el caso de Turisme de Catalunya, que ha publicado la guía Turismo LGBT. Esta guía identifica la oferta existente en las diez marcas turísticas catalanas específica para el colectivo LGBT. Paralelamente a la publicación de la guía, Turisme de Catalunya también ha puesto en marcha una nueva página web sobre este tipo de turismo (http://catalunya-lgbt.catalunya.com/ca), donde, además de ofrecer la posibilidad de descargar la guía, se informa sobre la oferta de turismo gay, lésbico, bisexual y transexual existente en todo el territorio catalán. Para ello está organizada en dos ejes: el temático y el territorial. En el eje temático, el usuario encuentra los recursos distribuidos por categorías, como "playas", «compras» o "para salir», entre otras. En el eje territorial se informa, igual que en la publicación en papel, de la oferta distribuida en las diez marcas territoriales catalanas. A través de estos dos ejes el usuario puede realizar búsquedas o localizar los recursos que más se adapten a sus necesidades y que estén más próximos. Asimismo, la página web incorpora un área de propuestas y una agenda con los eventos específicos del sector LGBT que se desarrollan en Cataluña, así como un área de noticias en la que Turisme de Catalunya informa sobre las acciones promocionales que realiza relacionadas con este colectivo.

Desde el ámbito privado, en la ciudad de Barcelona se ofrece un sinfín de propuestas para el colectivo LGBT. De hecho, se ha constituido el denominado 
«Gaixample», que ocupa un tramo de la parte izquierda del Eixample, desde la calle de Aribau hasta la calle del Comte d'Urgell, y que acoge muchos establecimientos pensados para uso y disfrute exclusivo de este colectivo. Este «metabarrio» (un barrio dentro de otro barrio) no es un gueto (área separada para la vivienda de un determinado grupo étnico, cultural o religioso, voluntaria o involuntariamente, en mayor o menor reclusión), ya que en el barrio viven y confluyen comercios y vecinos de todo tipo de tendencias, incluye una amplia variedad de opciones, desde discotecas, bares y clubs de ambiente, agencias de viajes especializadas y tiendas de ropa o de cosméticos específicas para su estilo hasta hoteles exclusivos, como son los casos del Hotel Axel, en la misma calle de Aribau, el GoGay Barcelona, en la calle de Balmes, o el Beauty and the Beach Gay, ya en el barrio del Poblenou.

Este fenómeno no ha sido ajeno al impulso dado gracias a la realización de eventos recreativos anuales de gran magnitud, como el Pride Barcelona y el Circuit Festival. El Pride Barcelona es un festival que se celebra durante una semana, a finales de junio, que ha atraído a más de 250.000 visitantes en el año 2014, con un gasto superior a los 20 millones de euros. Se trata de un conjunto de más de cien actividades culturales y de ocio (conciertos y actuaciones musicales, ferias gastronómicas y comerciales, debates, actividades deportivas, cabalgatas, la elección de una "miss», la fiesta de la espuma y múltiples juegos). Para ello se cuenta con el patrocinio de grandes empresas multinacionales, treinta asociaciones, la colaboración de los organismos públicos catalanes (Generalitat de Catalunya, Diputación de Barcelona, Ayuntamiento de Barcelona), de la Asociación Empresarial PIMEC y de Transportes Municipales de Barcelona (TMB). Entre los asistentes, más del $80 \%$ son extranjeros, principalmente alemanes, británicos y estadounidenses.

El Festival Circuit es uno de los principales eventos europeos de ocio y turismo cultural pensado para el disfrute del colectivo LGBT de todas las edades y condiciones, con más de 70.000 participantes en 2014 (Figura 1) que han generando unos ingresos superiores a los 100 millones de euros. En él se

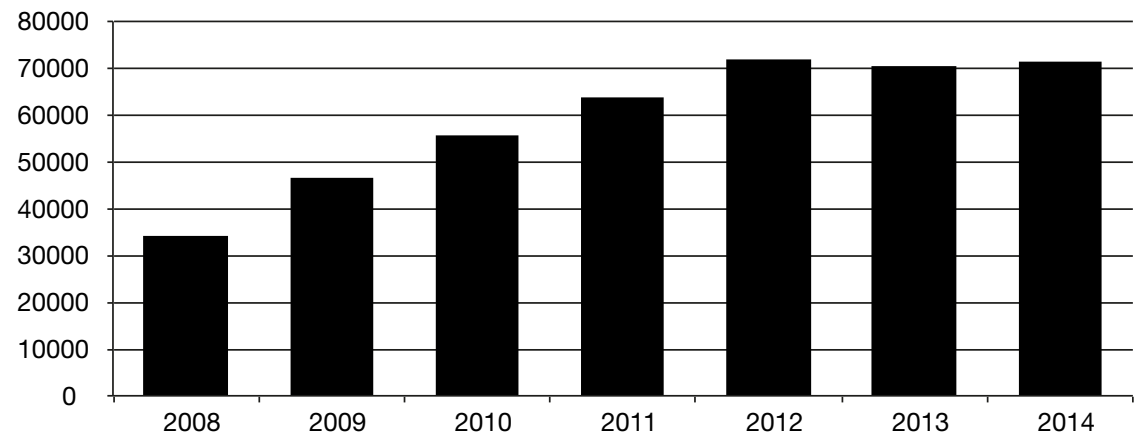

Figura 1. Evolución de visitantes al Festival Circuit.

Fuente: Matinée Group, 2014. 
pueden realizar múltiples actividades sociales, culturales y de ocio (Prat, 2014). Como en el caso anterior, aquí también casi el $80 \%$ de los participantes son de procedencia extranjera, con predominio de franceses, italianos y británicos, aunque en estas últimas ediciones también han aumentado considerablemente los brasileños, rusos, indios y estadounidenses. Según datos facilitados por Matinée Group (2012) —entidad organizadora del Festival—, el perfil de los asistentes es de hombres y mujeres menores de 40-45 años, profesionales liberales, con estudios medios o superiores y clase social media-alta y alta, lo que les permite disponer de una buena capacidad de consumo.

\section{Metodología}

Para comprobar las hipótesis aquí planteadas se han analizado los resultados de dos encuestas realizadas en conjunto a 116 turistas LGBT, con un error muestral máximo del $9 \%$, para un intervalo de confianza de $\pm 95 \%$ y una heterogeneidad del $50 \%$. La primera fue realizada personalmente por el autor de este artículo, mediante un cuestionario cerrado, a un grupo de asistentes al Festival Circuit de Barcelona. Dicha encuesta se hizo en agosto de 2012 a una muestra de 62 turistas LGBT extranjeros que asistían a este festival, escogidos al azar. El error muestral fue del $12,5 \%$, con un intervalo de confianza de $\pm 95 \%$ y una heterogeneidad del $50 \%$. La segunda fue realizada por el mismo autor en otoño de 2014 a otros 54 turistas LGBT, sin tener en cuenta su lugar de procedencia, que estaban en aquel momento en la ciudad pero que no habían asistido nunca al Festival Circuit. El error muestral fue del 13,5\%, con un intervalo de confianza de $\pm 95 \%$ y una heterogeneidad del $50 \%$. En ambos casos se utilizó un cuestionario con las mismas preguntas.

En la Tabla 1 se presenta el perfil de los encuestados en ambas muestras. Para el diseño del cuestionario se ha partido del modelo de Prat y Cànoves (2012) sobre la motivación de los turistas LGBT en la elección de destinos turísticos urbanos y de las preguntas diseñadas por Prat (2014) para analizar las motivaciones de los turistas LGBT en la elección del Festival Circuit. En este modelo, a pesar de considerarse que las motivaciones que guían al turista pueden ser muy variadas (McKercher y Du Cros, 2003), se identificaron varios grupos de factores aplicables específicamente al turismo LGBT, siguiendo otros estudios anteriores (Molina et al., 2007; Mondéjar y Gómez, 2009). Unos estaban relacionados con las motivaciones del turista. Otros eran relativos al binomio calidad-precio de los servicios turísticos. El principal motivo para elegir este modelo fue que es el más utilizado en la elección de un destino turístico, ya que tiene en cuenta la motivación por la satisfacción de diferentes necesidades personales (Sirakaya y Woodside, 2005; Prat 2014).

En dicho modelo hay tres grandes grupos de variables: intrínsecas, cualitativas y pragmáticas (Prat, 2014). Las intrínsecas comprenden los aspectos afectivos (experiencias anteriores / relaciones personales / recomendaciones) y las nuevas vivencias (estar de moda / aumentar la red de relaciones personales / 
Tabla 1. Perfil de los participantes en las encuestas

\begin{tabular}{|c|c|c|c|c|}
\hline Género & \multicolumn{2}{|c|}{$\begin{array}{l}\text { Con Festival } \\
\text { Circuit (1) }\end{array}$} & \multicolumn{2}{|c|}{$\begin{array}{l}\text { Sin Festival } \\
\text { Circuit (2) }\end{array}$} \\
\hline Hombres & 35 & $56,45 \%$ & 31 & $57,41 \%$ \\
\hline Mujeres & 27 & $43,55 \%$ & 23 & $42,59 \%$ \\
\hline \multicolumn{5}{|l|}{ Grupo de edad } \\
\hline$<30$ años & 18 & $29,03 \%$ & 10 & $18,52 \%$ \\
\hline 30-45 años & 29 & $46,77 \%$ & 23 & $42,59 \%$ \\
\hline $45-60$ años & 11 & $17,74 \%$ & 14 & $25,93 \%$ \\
\hline$>60$ años & 4 & $6,45 \%$ & 7 & $12,96 \%$ \\
\hline \multicolumn{5}{|l|}{ Nivel de estudios } \\
\hline Elementales & 8 & $12,90 \%$ & 5 & $9,26 \%$ \\
\hline Medios & 29 & $46,77 \%$ & 29 & $53,70 \%$ \\
\hline Superiores & 25 & $40,32 \%$ & 20 & $37,04 \%$ \\
\hline \multicolumn{5}{|l|}{ Profesión } \\
\hline Funcionario & 4 & $6,45 \%$ & 2 & $3,70 \%$ \\
\hline Liberal & 19 & $30,65 \%$ & 17 & $31,48 \%$ \\
\hline Autónomo & 14 & $22,58 \%$ & 12 & $22,22 \%$ \\
\hline Contratado & 12 & $19,35 \%$ & 9 & $16,67 \%$ \\
\hline Estudiante & 5 & $8,06 \%$ & 3 & $5,56 \%$ \\
\hline Sin trabajo específico & 8 & $12,90 \%$ & 11 & $20,37 \%$ \\
\hline \multicolumn{5}{|l|}{ Nivel económico } \\
\hline Bajo & 2 & $3,23 \%$ & 1 & $1,85 \%$ \\
\hline Medio-bajo & 8 & $12,90 \%$ & 6 & $11,11 \%$ \\
\hline Medio & 33 & $53,23 \%$ & 28 & $51,85 \%$ \\
\hline Medio-alto & 15 & $24,19 \%$ & 17 & $31,48 \%$ \\
\hline Alto & 4 & $6,45 \%$ & 2 & $3,70 \%$ \\
\hline
\end{tabular}

Fuentes: (1) = Matinée Group, 2012 y (2) = elaboración propia, 2014.

curiosidad / conocer una nueva ciudad). Las cualitativas están formadas por el punto de vista del turista sobre la oferta (organización del evento / actividades culturales / actividades recreativas y de ocio) y sobre el lugar (imagen global percibida de la ciudad / información adquirida sobre el destino / posibles actividades en la ciudad aparte de las previstas en la oferta). Las pragmáticas tienen en cuenta el precio de la oferta / calidad de la oferta / información sobre la oferta / coste del viaje / disponibilidad de fechas (Prat, 2014). Todos estos grupos se encuentran interrelacionados, por lo que influyen en la elección del destino, que es la variable endógena del modelo (Prat y Cànoves, 2012).

Para ello se diseñaron treinta preguntas, teniendo en cuenta que las respuestas podían ir de 1 (menos) a 5 (más), según la escala de Lickert, sobre diversos aspectos para elegir la ciudad de Barcelona como destino turístico del colectivo LGBT. De estas preguntas, tres incidían en el aspecto afectivo, cuatro en el pragmático, dieciséis tenían en cuenta las cualidades propias del lugar visitado, 
tres consideraban las cualidades del propio evento y las cuatro restantes estaban relacionadas con la posibilidad de vivir nuevas experiencias.

Una vez realizadas y codificadas ambas encuestas, los resultados obtenidos fueron sometidos a un análisis estadístico informático para la valoración media de cada grupo de variables para ambas muestras, comparando las medias obtenidas para cada variable con el objetivo de comprobar las hipótesis propuestas. El programa utilizado fue el StatGraphics Centurion.

Finalmente, es importante tener en cuenta que en el análisis de las encuestas se presentan varias limitaciones. La primera se refiere a la medición de las variables antes y después de la experiencia turística en un mismo momento. Así, por cuestiones prácticas, la primera encuesta se realizó antes de que el turista LGBT hubiese participado en el Festival Circuit, lo que puede condicionar las relaciones entre las variables pre y postexperiencia y, además, provocar que los juicios «a priori» estén sujetos a ciertas pérdidas de memoria o distorsión de la información en su recuperación. La segunda se refiere al uso de las expectativas predictivas, que, si bien son el estándar de comparación más utilizado en el proceso global de satisfacción, han recibido crecientes críticas en la literatura académica al respecto (Yüksel y Yüksel, 2001). Estas críticas se refieren al hecho de que los turistas podrían no tener la motivación suficiente como para generar expectativas para ciertas clases de productos y, además, pueden utilizar diferentes estándares dependiendo de ciertos factores personales y del contexto. La tercera limitación es el espacio geográfico donde se ha desarrollado la investigación, ya que el destino turístico objeto de examen (la ciudad de Barcelona) condiciona los atributos que deben incluirse dentro de la metodología estructurada de medición de la imagen y, además, determina las propiedades de la muestra. Sin embargo, a pesar de estas limitaciones, no hay que olvidar que las normas basadas en la experiencia son quizás el mejor estándar, ya que recogen la experiencia de diferentes grupos de turistas LGBT. La cuarta es el reducido tamaño muestral de ambas encuestas, lo que aumenta su margen de error.

\section{Resultados}

Los resultados obtenidos en ambas encuestas para cada una de las variables presentadas en el cuestionario son los que se detallan en el siguiente cuadro (Tabla 2), teniendo en cuenta que el valor máximo es cinco.

En la tabla 2 se puede observar que los motivos más valorados por los turistas LGBT cuya motivación principal para viajar a Barcelona era asistir al Festival Circuit fueron las ganas de divertirse $(4,65)$, su grado de satisfacción por la experiencia de años anteriores $(4,48)$, la imagen de la ciudad $(4,48)$, la posibilidad de volver a ver a los amigos $(4,36)$, el clima de la ciudad $(4,22)$, el patrimonio urbano $(4,17)$ y las múltiples actividades recreativas ofertadas en el destino $(4,16)$. A su vez, los aspectos menos valorados fueron hacer la visita por estar de moda (2,95), la señalización de la ciudad $(2,86)$ y la curiosidad por realizar esta experiencia $(2,85)$.

Los factores que más valoraron los LGBT que no asistieron a este macroevento son su satisfacción por los viajes anteriores a la ciudad $(4,87)$, ver 
Tabla 2. Resultado de las encuestas

\begin{tabular}{|c|c|c|c|c|}
\hline \multirow[b]{2}{*}{ Variables analizadas } & \multicolumn{2}{|c|}{$\begin{array}{c}\text { Con Festival } \\
\text { Circuit (1) }\end{array}$} & \multicolumn{2}{|c|}{$\begin{array}{l}\text { Sin Festival } \\
\text { Circuit (2) }\end{array}$} \\
\hline & $\begin{array}{c}\text { Valoración } \\
\text { media por } \\
\text { variable }\end{array}$ & $\begin{array}{c}\text { Valoración } \\
\text { media por } \\
\text { grupo }\end{array}$ & $\begin{array}{c}\text { Valoración } \\
\text { media por } \\
\text { variable }\end{array}$ & $\begin{array}{l}\text { Valoración } \\
\text { media por } \\
\text { grupo }\end{array}$ \\
\hline Aspectos afectivos & & 4,32 & & 4,69 \\
\hline Ver amigos & 4,36 & & 4,79 & \\
\hline Recomendaciones de amigos & 4,13 & & 4,42 & \\
\hline Satisfacción de viajes anteriores & 4,48 & & 4,87 & \\
\hline Cualidades de la oferta & & 3,63 & & 2,96 \\
\hline Precio & 3,21 & & 2,85 & \\
\hline Información turística disponible & 3,37 & & 2,27 & \\
\hline Publicidad & 3,92 & & 2,34 & \\
\hline Calidad del alojamiento & 4,03 & & 4,36 & \\
\hline Cualidades del lugar & & 3,65 & & 4,04 \\
\hline Señalización de la ciudad & 2,86 & & 2,68 & \\
\hline Control del ruido medioambiental & 3,04 & & 3,36 & \\
\hline Disponibilidad de transportes urbanos & 3,08 & & 3,95 & \\
\hline Limpieza de la ciudad & 3,15 & & 3,93 & \\
\hline Infraestructuras y equipamientos & 3,44 & & 4,36 & \\
\hline Servicios existentes & 3,31 & & 4,39 & \\
\hline Hospitalidad de la comunidad local & 3,47 & & 4,49 & \\
\hline Conservación del entorno & 3,57 & & 3,95 & \\
\hline Visitas culturales & 3,85 & & 3,94 & \\
\hline Cercanía de la playa & 3,93 & & 4,73 & \\
\hline Seguridad ciudadana & 3,87 & & 4,45 & \\
\hline Clima & 4,22 & & 4,52 & \\
\hline Gastronomía local & 4,04 & & 4,05 & \\
\hline Facilidad del acceso a la ciudad & 3,97 & & 3,91 & \\
\hline Patrimonio urbano & 4,17 & & 4,34 & \\
\hline Imagen de la ciudad & 4,48 & & 3,53 & \\
\hline Aspectos pragmáticos & & 4,31 & & 3,57 \\
\hline Organización de los eventos & 4,13 & & 2,54 & \\
\hline Actividades recreativas & 4,16 & & 4,03 & \\
\hline Diversiones & 4,65 & & 4,14 & \\
\hline Nuevas experiencias & & 3,39 & & 2,89 \\
\hline Curiosidad & 2,85 & & 2,23 & \\
\hline Estar de moda & 2,95 & & 3,08 & \\
\hline Nuevas experiencias & 3,69 & & 2,61 & \\
\hline Conocer nuevos amigos & 4,06 & & 3,62 & \\
\hline
\end{tabular}

Fuentes: (1) = Matinée Group, 2012 y (2) = elaboración propia, 2014. 
a los amigos $(4,79)$, la posibilidad de ir fácilmente a la playa $(4,73)$ y el clima $(4,52)$. Los aspectos menos valorados por este colectivo son la información turística disponible $(2,27)$, la publicidad sobre las posibilidades turísticas de la ciudad $(2,34)$ y la curiosidad por conocerla turísticamente $(2,23)$.

Agrupando los resultados según el modelo aquí aplicado, los aspectos más valorados por el colectivo LGBT que asistió al festival Circuit son los factores afectivos y los pragmáticos, mientras que los menos valorados son las nuevas experiencias. Por su parte, los LGBT que no acudieron a este macroevento también valoraron mejor los aspectos afectivos, mientras que las cualidades de la oferta y las nuevas experiencias fueron las peor valoradas. Así pues, ambos colectivos de LGBT consideran muy importantes para su elección del destino los aspectos afectivos (volver a estar con los amigos, experiencias satisfactorias de viajes anteriores), y lo que peor valoran es el consumo de nuevas experiencias (curiosidad, moda, nuevos conocimientos). Por el contrario, una diferencia entre ambos grupos es que los primeros también valoran muy positivamente los aspectos pragmáticos (diversiones, eventos, atracciones), mientras que los segundos valoran más negativamente las cualidades de la oferta (información, precio, calidad).

Variable por variable, la comparación entre ambas muestras resalta que las mayores diferencias en la valoración (más de un punto y medio sobre cinco) se encuentran en la publicidad y en la organización de eventos, ambas muy bien consideradas por los que asistieron al Festival Circuit y muy poco por los que no. A su vez, los factores cuya valoración es muy similar en ambos colectivos son los correspondientes a la gastronomía local, la facilidad de acceso, la señalización en la ciudad, el patrimonio existente, las visitas culturales, las actividades recreativas y la moda.

Aplicando los resultados de la valoración media por grupo para ambas muestras al programa estadístico StatGraphics, se ha obtenido la Tabla 3.

Esta tabla muestra el resumen estadístico para cada uno de los grupos de variables seleccionadas. De particular interés es el sesgo estandarizado y la curtosis estandarizada, que pueden usarse para determinar si la muestra proviene de una distribución normal. Valores de estos estadísticos fuera del rango de -2 $\mathrm{a}+2$ indican desviaciones significativas de la normalidad, las cuales tenderían a invalidar muchos de los procedimientos estadísticos que se aplican habitualmente a estos datos. En el caso presente no hay variables con valores de sesgo estandarizado y de curtosis estandarizada fuera del rango esperado.

A continuación, para validar las hipótesis aquí propuestas, se han analizado y comparado los resultados y las medias de cada muestra para cada uno de los grupos de variables de forma individual.

\section{a) Aspectos afectivos}

En la Tabla 4 se presenta el resumen estadístico del primer grupo de variables analizado para las dos muestras de datos («aspectos afectivos»). En este caso, no se observan valores de sesgo estandarizado y de curtosis estandarizada 
Tabla 3. Resumen estadístico de cada aspecto analizado

\begin{tabular}{lrrrrr}
\hline & $\begin{array}{r}\text { Aspectos } \\
\text { afectivos }\end{array}$ & $\begin{array}{c}\text { Cualidades } \\
\text { de la oferta }\end{array}$ & $\begin{array}{c}\text { Cualidades } \\
\text { del lugar }\end{array}$ & $\begin{array}{c}\text { Aspectos } \\
\text { pragmáticos }\end{array}$ & $\begin{array}{c}\text { Nuevas } \\
\text { experiencias }\end{array}$ \\
\hline Recuento & 116 & 116 & 116 & 116 & 116 \\
Promedio & 4,49138 & 3,31897 & 3,82759 & 3,96552 & 3,15517 \\
Mediana & 5,0 & 3,0 & 4,0 & 4,0 & 3,0 \\
Moda & 5,0 & 3,0 & 4,0 & 4,0 & 3,0 \\
Varianza & 0,391229 & 0,619115 & 0,387406 & 0,694453 & 0,601799 \\
Desviación estándar & 0,625483 & 0,786839 & 0,62242 & 0,833338 & 0,775757 \\
Coeficiente de variación & $13,9263 \%$ & $23,7074 \%$ & $16,2614 \%$ & $21,0146 \%$ & $24,5868 \%$ \\
Error estándar & 0,0580747 & 0,0730562 & 0,0577902 & 0,0773735 & 0,0720272 \\
Mínimo & 3,0 & 2,0 & 3,0 & 2,0 & 2,0 \\
Máximo & 5,0 & 5,0 & 5,0 & 5,0 & 5,0 \\
Rango & 2,0 & 3,0 & 2,0 & 3,0 & 3,0 \\
Cuartil inferior & 4,0 & 3,0 & 3,0 & 3,0 & 3,0 \\
Cuartil superior & 5,0 & 4,0 & 4,0 & 5,0 & 4,0 \\
Sesgo estandarizado & $-1,97043$ & 0,0883514 & 0,587062 & $-1,73$ & $-0,720889$ \\
Curtosis estandarizada & $-0,660831$ & $-1,0474$ & $-1,07285$ & $-1,09617$ & $-1,96069$ \\
\hline
\end{tabular}

Fuente: elaboración propia con StatGraphics.

Tabla 4. Resumen estadístico de la variable «aspectos afectivos»

\begin{tabular}{lrc}
\hline & Aspectos afectivos 1 & Aspectos afectivos 2 \\
\hline Recuento & 62 & 54 \\
Promedio & 4,32258 & 4,68519 \\
Desviación estándar & 0,672022 & 0,507457 \\
Coeficiente de variación & $15,5468 \%$ & $10,8311 \%$ \\
Varianza & 0,451613 & 0,257512 \\
Gl & 61 & 53 \\
Mínimo & 3,0 & 3,0 \\
Máximo & 5,0 & 5,0 \\
Rango & 2,0 & 2,0 \\
Sesgo estandarizado & $-1,56833$ & $-1,78984$ \\
Curtosis estandarizada & $-1,16153$ & 0,846975 \\
\hline
\end{tabular}

Fuente: elaboración propia con StatGraphics.

fuera del rango esperado. Seguidamente se han construido los intervalos de confianza para cada media y para la diferencia entre las medias y se ha ejecutado una prueba t para comparar las medias de las dos muestras. Los intervalos de confianza del 95,0\% para la media de «aspectos afectivos 1 " son 4,32258+/-0,170662, es decir [4,15192; 4,49324]. Los de «aspectos afectivos $2 », 4,68519+/-0,138509$, [4,54668; 4,82369]. Los de la diferencia de medias, suponiendo varianzas iguales, son $-0,362605+/-0,221665$, es decir $[-0,58427 ;-0,140939]$. Asimismo, la prueba t para comparar las medias, suponiendo varianzas iguales, ha dado como resultado: $\mathrm{t}=-3,24055$ 
$\mathrm{y}$ valor- $\mathrm{P}=0,00156398$. Puesto que el valor-P calculado es menor que $0,05 \mathrm{y}$ el intervalo de la diferencia de las medias no contiene el valor 0 , se rechaza la hipótesis nula (media $1=$ media 2 ) con un nivel de confianza del 95,0\%. Así pues, hay diferencias significativas entre estos dos grupos de variables.

\section{b) Cualidades de la oferta}

En este caso (Tabla 5), para el segundo grupo de variables («cualidades de la oferta»), ambos valores de sesgo estandarizado y de curtosis estandarizada se encuentran dentro del rango esperado. Si se comparan las medias de las dos muestras, los resultados indican que los intervalos de confianza del 95,0\% para la media de "cualidades de la oferta 1 » son 3,62903+/-0,173486, es decir $[3,45555 ; 3,80252]$. Los de la media de "cualidades de la oferta 2», 2,96296 +/-0,2051, [2,75786; 3,16806]. Los de la diferencia de medias, suponiendo varianzas iguales, $0,666069+/-0,263907$, es decir $[0,402163$; $0,929976]$. Además, la prueba t para comparar medias, suponiendo varianzas iguales, da como resultado: $\mathrm{t}=4,9998$ y valor- $\mathrm{P}=0,00000209652$. Puesto que el intervalo de la diferencia de medias no contiene el valor 0 y el valor-P calculado es menor que 0,05 , existe una diferencia estadísticamente significativa entre las medias de las dos muestras, con un nivel de confianza del $95,0 \%$ y, por tanto, se rechaza la hipótesis nula (media $1=$ media 2 ) para alfa $=0,05$. Así pues, también hay diferencias significativas entre estos dos grupos de variables.

Tabla 5. Resumen estadístico de la variable «cualidades de la oferta»

\begin{tabular}{lrc}
\hline & Cualidades de la oferta $\mathbf{1}$ & Cualidades de la oferta 2 \\
\hline Recuento & 62 & 54 \\
Promedio & 3,62903 & 2,96296 \\
Desviación estándar & 0,683143 & 0,751425 \\
Coeficiente de variación & $18,8244 \%$ & $25,3606 \%$ \\
Varianza & 0,466684 & 0,56464 \\
Gl & 61 & 53 \\
Mínimo & 2,0 & 2,0 \\
Máximo & 5,0 & 5,0 \\
Rango & 3,0 & 3,0 \\
Sesgo estandarizado & $-0,026127$ & 1,01521 \\
Curtosis estandarizada & $-0,250381$ & $-0,507515$ \\
\hline
\end{tabular}

Fuente: elaboración propia con StatGraphics.

\section{c) Cualidades del lugar}

Tal como se aprecia en el cuadro siguiente (Tabla 6), para este tercer grupo de variables («cualidades del lugar») ambos valores de sesgo estandarizado y de 
Tabla 6. Resumen estadístico de la variable «cualidades del lugar»

\begin{tabular}{lrc}
\hline & Cualidades del lugar $\mathbf{1}$ & Cualidades del lugar 2 \\
\hline Recuento & 62 & 54 \\
Promedio & 3,64516 & 4,03704 \\
Desviación estándar & 0,546127 & 0,643193 \\
Coeficiente de variación & $14,9822 \%$ & $15,9323 \%$ \\
Varianza & 0,298255 & 0,413697 \\
Gl & 61 & 53 \\
Mínimo & 3,0 & 3,0 \\
Máximo & 5,0 & 5,0 \\
Rango & 2,0 & 2,0 \\
Sesgo estandarizado & 0,0459198 & $-0,0969695$ \\
Curtosis estandarizada & $-1,32051$ & $-0,713463$ \\
\hline
\end{tabular}

Fuente: elaboración propia con StatGraphics.

curtosis estandarizada se encuentran dentro del rango esperado. Comparando las medias de ambas muestras, los intervalos de confianza del 95,0\% para la media de «cualidades del lugar 1 » son 3,64516 +/-0,138691, es decir [3,50647; $3,78385]$. Los de la media de "cualidades del lugar 2 » son 4,03704 +/- 0,175558, $[3,86148 ; 4,2126]$. Los de la diferencia de medias, suponiendo varianzas iguales, $-0,391876+/-0,218749$, es decir $[-0,610624 ;-0,173127]$. Puesto que el intervalo no contiene el valor 0 , existe una diferencia estadísticamente significativa entre las medias de las dos muestras, con un nivel de confianza del 95,0\%. A su vez, la prueba t para comparar medias, suponiendo varianzas iguales, da como resultado: $\mathrm{t}=-3,54884$ y valor- $\mathrm{P}=0,000563113(<0,05)$. Por tanto, se rechaza la hipótesis nula (media $1=$ media 2 ) para alfa $=0,05$. De este modo, también hay diferencias significativas entre estos dos grupos de variables.

\section{d) Aspectos pragmáticos}

En este caso (Tabla 7), para el cuarto grupo de variables («aspectos pragmáticos») ambos valores de sesgo estandarizado y de curtosis estandarizada se encuentran dentro del rango esperado. Si se comparan las medias de las dos muestras, los intervalos de confianza del 95,0\% para «aspectos pragmáticos 1» son 4,30645+/-0,169509, es decir [4,13694; 4,47596]. Los de «aspectos pragmáticos 2» son 3,57407 +/- 0,228684, [3,34539; 3,80276]. Los de la diferencia de medias, suponiendo varianzas iguales, son $0,732378+/-0,277107$, es decir $[0,455271 ; 1,00948]$. Puesto que el intervalo no contiene el valor 0 , existe una diferencia estadísticamente significativa entre las medias de las dos muestras, con un nivel de confianza del 95,0\%. A su vez, la prueba t para comparar medias, suponiendo varianzas iguales, da como resultado: $t=5,23567 \mathrm{y}$ valor-P $=7,58997 \mathrm{E}-7(<0,5)$. Por tanto, se rechaza la hipótesis nula (media 1 $=$ media 2) para alfa $=0,05$. Aquí también hay diferencias significativas entre estos dos grupos de variables. 
Tabla 7. Resumen estadístico de la variable «aspectos pragmáticos»

\begin{tabular}{lrc}
\hline & Aspectos pragmáticos 1 & Aspectos pragmáticos 2 \\
\hline Recuento & 62 & 54 \\
Promedio & 4,30645 & 3,57407 \\
Desviación estándar & 0,667481 & 0,837829 \\
Coeficiente de variación & $15,4996 \%$ & $23,4418 \%$ \\
Varianza & 0,445531 & 0,701957 \\
Gl & 61 & 53 \\
Mínimo & 3,0 & 2,0 \\
Máximo & 5,0 & 5,0 \\
Rango & 2,0 & 3,0 \\
Sesgo estandarizado & $-1,42277$ & $-0,128876$ \\
Curtosis estandarizada & $-1,16304$ & $-0,74414$ \\
\hline
\end{tabular}

Fuente: elaboración propia con StatGraphics.

\section{e) Nuevas experiencias}

Como se puede apreciar en el cuadro siguiente (Tabla 8), para este quinto grupo de variables ("nuevas experiencias») ambos valores de sesgo estandarizado y de curtosis estandarizada se encuentran dentro del rango esperado. Comparando las medias, se obtiene que los intervalos de confianza del 95,0\% para la media de «nuevas experiencias 1 " son 3,3871 +/-0,174222, es decir [3,21287; $3,56132]$. Para la media de «nuevas experiencias 2 » son $2,88889+/-0,216462$, $[2,67243 ; 3,10535]$ y para la diferencia de medias, suponiendo varianzas iguales, $0,498208+/-0,272028,[0,22618 ; 0,770236]$. Puesto que el intervalo no contiene el valor 0 , existe una diferencia estadísticamente significativa entre las medias de las dos muestras, con un nivel de confianza del 95,0\%. A su vez, la prueba t para comparar medias, suponiendo varianzas iguales, da como resultado: $\mathrm{t}=3,62811$ y valor- $\mathrm{P}=0,00042863(<0,5)$. Por tanto, se rechaza la hipótesis nula (media $1=$ media 2 ) para alfa $=0,05$. De esta manera, también hay diferencias significativas entre estos dos grupos de variables.

En consecuencia, la comparación entre las dos muestras indica que existen diferencias significativas entre ambos colectivos en todos los cinco grupos de variables que influyen en la elección de los destinos por parte de los turistas LGBT (H1). Además, el aspecto más valorado por los que realizan el viaje sin el reclamo de un gran evento LGBT es el afectivo (H2), y destaca la satisfacción obtenida en anteriores viajes a este destino y la posibilidad de reencontrarse con los amigos. Finalmente, el aspecto lúdico predomina en la elección del destino por parte de los turistas que vienen a Barcelona para asistir a un macroevento preparado para el colectivo LGBT (H3).

En cuanto al lugar, la percepción manifestada por ambos colectivos es distinta, y es más favorable en conjunto en el caso de los segundos, los cuales valoran más positivamente aspectos como la hospitalidad de los residentes, los servicios y las infraestructuras existentes en el destino y la seguridad. En cambio, ambos colectivos aprecian factores como el clima, la playa, la oferta 
Tabla 8. Resumen estadístico para la variable «nuevas experiencias»

\begin{tabular}{lrc}
\hline & Nuevas experiencias 1 & Nuevas experiencias 2 \\
\hline Recuento & 62 & 54 \\
Promedio & 3,3871 & 2,88889 \\
Desviación estándar & 0,68604 & 0,793052 \\
Coeficiente de variación & $20,2545 \%$ & $27,4518 \%$ \\
Varianza & 0,47065 & 0,628931 \\
Gl & 61 & 53 \\
Mínimo & 2,0 & 2,0 \\
Máximo & 5,0 & 4,0 \\
Rango & 3,0 & 2,0 \\
Sesgo estandarizado & $-1,16984$ & 0,611077 \\
Curtosis estandarizada & $-0,666769$ & $-1,95585$ \\
\hline
\end{tabular}

Fuente: elaboración propia con StatGraphics.

gastronómica y el patrimonio cultural. Por el contrario, los primeros tienen más en cuenta la imagen de la ciudad en el momento de la elección del destino.

\section{Conclusiones}

Barcelona y el turismo han ido siempre de la mano. Los viajeros siempre han llegado a su puerto y han paseado por sus plazas y calles, han comprado en sus tiendas y se han deleitado con las múltiples actividades que allí se realizaban. Los Juegos Olímpicos de 1992 sirvieron para abrir la ciudad al mar, mejorar sus infraestructuras y, sobre todo, darla a conocer internacionalmente.

De esta manera, con una eficiente promoción institucional, se ha desarrollado el turismo urbano como elemento clave en el crecimiento económico de la ciudad y de la región. El turismo LGBT no ha sido ajeno a este fenómeno. Sin embargo, al tratarse de un colectivo en el que coinciden grupos de personas con diferentes motivaciones e intereses, es necesario tener estas muy en cuenta para diseñar un plan promocional efectivo de la ciudad.

Se trata de un segmento interesante y rentable para las empresas del sector turístico, por su poder adquisitivo, frecuencia de viajes y nivel de consumo. Sin embargo, no todo el turismo LGBT se ciñe a este modelo, sino que estos turistas pueden llegar a convertirse en clientes habituales de un determinado destino (en nuestro caso, la ciudad de Barcelona), si este logra presentarle una oferta mejor que la competencia en la satisfacción de sus motivaciones, necesidades y expectativas personales y sociales en la elección del viaje.

Además, la imagen percibida del destino antes de realizar el viaje tiene un gran componente de naturaleza multidimensional y, en consecuencia, los turistas LGBT utilizarán las distintas dimensiones de la imagen para comparar los destinos evocados en su mente una vez surgida la motivación de viajar y seleccionar el destino turístico. De este modo, para promocionar eficazmente un destino turístico no se deben enfatizar exclusivamente los recursos natura- 
les o culturales del lugar, sino también las emociones y sentimientos que este puede evocar.

Por ello, en este artículo, después de confirmarse la importancia de la ciudad de Barcelona como emergente destino para el turismo LGBT internacional, con el apoyo de las instituciones públicas y privadas, se ha visto que los diversos factores - afectivos, cualitativos y pragmáticos — analizados a partir de las dos encuestas realizadas confirman que la motivación de los turistas LGBT en la elección de un determinado destino urbano viene condicionada por sus preferencias iniciales para realizar el viaje. En estas predomina el aspecto lúdico y festivo de la visita en el caso de los que vienen para asistir a un macroevento pensado específicamente para este colectivo y el aspecto afectivo en el caso de los que no acuden por esta circunstancia.

La comparación de los resultados de las encuestas realizadas a una muestra de turistas LGBT que visitan Barcelona atraídos por un macroevento pensado específicamente para este colectivo, como el Festival Circuit, y los que no, confirma la existencia de diferencias significativas entre las motivaciones de ambos colectivos en la elección de este destino urbano. El aspecto más valorado por los primeros es el lúdico, mientras que el afectivo lo es en el caso de los segundos. También del análisis de ambos casos se extrae la conclusión de que para este tipo de turismo no existe solamente una lógica, sino un conjunto de lógicas diferentes para elegir un determinado destino turístico urbano.

Dicha decisión tiene un gran componente emocional y afectivo, que, en muchas ocasiones, no se toma individualmente sino de manera conjunta, y si se toma individualmente afecta a la conducta de otros, teniendo en cuenta que la naturaleza de la experiencia buscada, tanto como las experiencias vividas en el pasado, marca la decisión, y que la experiencia está asociada más con el tipo de viaje que se lleva a cabo que con el lugar en el que este se realiza. Sin embargo, en la decisión de la elección también influyen otros factores, como la imagen de calidad que proyecta el destino y la calidad de la oferta de las actividades que allí se podrán realizar a lo largo de la estancia.

Ante esta situación, las organizaciones privadas y públicas que identifiquen las necesidades y expectativas de este tipo de demanda, no satisfechas por la oferta existente, tienen mejores oportunidades para atraer este turismo y desarrollar el negocio a su alrededor. De esta manera, tener el mayor conocimiento posible acerca de las necesidades de estos turistas y diseñar una oferta flexible, especializada y acorde a las mismas es sinónimo de obtener una ventaja competitiva en el posicionamiento de una ciudad como destino del turismo LGBT.

Finalmente, sería interesante que el presente trabajo pudiese completarse en el futuro con investigaciones similares en otras ciudades $\mathrm{u}$ otros grupos de este mismo colectivo, de manera que las conclusiones obtenidas pudieran generalizarse y servir de patrón en la promoción y desarrollo de la oferta de turismo LGBT. 


\section{Referencias bibliográficas}

Barrado, D. A. (2010). «Gran ciudad y turismo en la transición postindustrial: nuevos y viejos procesos, nuevas y viejas teorías. El ejemplo del área metropolitana de Madrid». Scripta Nova, vol. XIV, 317, 7-36.

Beddoe, C. (2003). "Aids and tourism: A deadly combination». En: T. Bauer T y B. Mc Kercher, (coed.). Sex and tourism, journeys of romance, love and lust. Nueva York: The Haworth Press, 197-207.

Benach, N. y Tello, R. (2004). «En los intersticios de la renovación. Estrategias de transformación del espacio y flujos de población en Barcelona». Revista de Geografía, 3, 93-114.

BORJA, J. (2010). Luces y sombras del urbanismo de Barcelona. Barcelona: UOC.

Buhalis, D. (2000). «Marketing the competitive destination of the future». Tourism Management, 21 (1), 97-116. <http://dx.doi.org/10.1016/S0261-5177(99)00095-3>

Cooper, D. (2010). "Theorising Nudist Equalirty: An Encounter Between Political Fantasy and Public Appearance». Antipode, 0 (00), 1-32.

Cresswell, T. (1996). In Place/Out of Plac: Geography, Ideology and Trangression. Minneapolis: University of Minnesota Press.

Edensor, T. (2001). «Performing tourism, staging tourism: (re)producing tourist space and practice». Tourist Studies, 1, 59-81. <http://dx.doi.org/10.1177/146879760100100104>

Fernández Salinas, V. (2007). "Visibilidad y escena gay masculina en la ciudad española». Documents d'Anàlisi Geogràfica, 49, 139-160.

Forest, B. (1995). "West Hollywood as symbol: the significance of place in the construction of a gay identity». Environment and Planning. Society and Space, 13 (2), 133-157. <http://dx.doi.org/10.1068/d130133>

Gibson, C. (2012). Geographies of tourism: space, ethics and encounter. <http://ro.uow. edu.au/cgi/viewcontent.cgi? article $=1568 \&$ context $=$ smhpapers $>$ [consulta: $11 \mathrm{de}$ marzo de 2015].

Gonzáles, F. y Morales, S. (2009). Ciudades efimeras: transformando el turismo urbano a través de la producción de eventos. Barcelona: UOC.

Hosteltur (2007). Noticias de turismo y economía. Madrid: Hosteltur.

Hughes, H. (2002). "Gay men's holiday destination choice: a case of risk and avoidance». International Journal of Tourism Research, 4, 299-312. <http://dx.doi.org/10.1002/jtr.382>

- (2006). Pink tourism: Holidays of gay men and lesbians. Oxford: Cabi. <http://dx.doi.org/10.1079/9781845930769.0000>

Jefrreys, S. (2003). «Sex tourism: Do women do it too?». Leisure Studies, 22, 223-238. <http://dx.doi.org/10.1080/026143603200075452>

Jensen-Campbell, C. (2004). The gay/lesbian travel marketplace. San Francisco: USDM.

JEYAsingham, D. (2011). «Building heteronormativity: the social and material reconstruction of men's public toilets as spaces of heterosexuality». Social \& Cultural Geography, 11 (4), 307-325. <http://dx.doi.org/10.1080/14649361003787706>

Knopp, L. (1998). «Sexuality and urban space: Gay male identity politics in the United States, the United Kingdom, and Australia». En: J. Jacobs, y R. Fincher, R. (eds.). Cities of Difference. Nueva York: The Guilford Press, 149-176. 
Martínez Quintana, V. (2006). Ocio y turismo en la sociedad actual. Los viajes, el tiempo libre y el entretenimiento en el mundo globalizado. Madrid: McGraw Hill.

Massey, D. (1994). Space, place and gender. Cambridge: Polity Press.

Massey, D.; Jess, P. y Massey, D. (1995). A Place in the World. Places, Cultures and Globalization. Oxford: Oxford University Press.

Mckercher, B. y Bauer, T. (2003). «Conceptual Framework of the Nexus between Tourism, Romance and Sex». En: T. Bauer,y B. Mc Kercher (coed.). Sex and tourism, journeys of romance, love and lust. Nueva York: The Haworth Press, 3-18.

Mckercher, B. y Du Cros, H. (2003). «Testing a cultural tourism typology». International Journal of Tourism Research, 5 (1), 45-58. $<$ http://dx.doi.org/10.1002/jtr.417>

Molina, A., Martín-Consuegra, D., Esteban, A. y Díaz, E. (2007). «Segmentación de la demanda turística. Un análisis aplicado a un destino de turismo cultural». Revista de Análisis Turístico, 4, 36-48.

Mondéjar, J. A. y Gómez, M. A. (2009). Turismo cultural en ciudades patrimonio de la humanidad. Cuenca: Ediciones de la Universidad de Castilla-La Mancha.

Moner, C.; Royo, M. y Ruiz, M. E. (2006). «El mercado turístico homosexual: un estudio para Cataluña». Estudios Turísticos, 167, 103-129.

Monterrubio, J. C. (2008). "Comunidades receptoras y percepciones: un estudio sobre turismo y sexualidad». Teoría y Praxis, 5, 145-160.

Norrild, J. (2007). "Relación entre turismo, género y sexo. El caso de Buzios-Brasil». Pasos. Revista de Turismo y Patrimonio Cultural, 5 (3), 331-341.

OMT (2014). Compendium of Tourism Statistics. Madrid: OMT. www2.unwto.org. [Consulta: 10 marzo 2015].

Oswin, N. (2006). "Decentering queer globalization: diffusion and the "global gay"». Environment and Planning. Society and Space, 24 (5), 777-790. $<$ http://dx.doi.org/10.1068/d63j>

Pino, A. (2010). «Educación afectivo-sexual y migración». Jornadas de Educación y Atención a la Sexualidad: Nuevos Retos. La Laguna (Tenerife), 23 y 24 de septiembre.

Prat, J. M. (2010). "Turismo naturista: una apuesta por el turismo marginal. El caso del barrio naturista de Cap d'Agde en Francia». Cuadernos de Turismo, 26, 207-222.

- (2014). «Nuevos planteamientos y nuevas motivaciones en la elección de destinos de turismo cultural-urbano homosexual: el Festival Circuit de Barcelona». Cuadernos de Turismo, 33, 311-334.

Prat, J. M. y Cànoves, G. (2012). "La motivación del turista homosexual en la elección de destinos culturales urbanos. Aplicación de un modelo de ecuaciones estructurales». Turydes, vol. 5, no 13 .

Pritchard, A. y Morgan, N. (2000). «Constructing tourism landscapes - gender, sexuality and space». Tourism Geographies, 2, 115-39. <http://dx.doi.org/10.1080/14616680050027851>

Pritchard, A.; Morgan, N.; Sedgely, D.; Khan, E. y Jenkins, A. (2000). «Sexuality and holiday choices: conversations with gay and lesbian tourists». Leisure Studies, 19 (4), 267-283.

<http://dx.doi.org/10.1080/02614360050118832>

Raibaud, Y. (2007). Le genre et le sexe comme objects géographiques. París: ADESCNRS.

Russell, P. (2001). «The world gay travel market». Travel \& Tourism Intelligence: Travel \& Tourism Analyst, 2, 37-57. 
Salazar, N. (2012). N. B. "Tourism imaginaries». Annals of Tourism Research, 39, 863-882. <http://dx.doi.org/10.1016/j.annals.2011.10.004>

Shankle, M. D. (2006). The Handbook of Lesbian, Gay, Bisexual, and Transgender Public Health: A Practitioner's Guide To Service. Nueva York: Harrington Park Press.

Sirakaya, E. y Woodside, A. G. (2005). «Building and testing theories of decision making by travellers». Tourism Management, 26, 815-832. <http://dx.doi.org/10.1016/j.tourman.2004.05.004>

SMith, A. (2012). Events and urban regeneration: the strategic use of events to revitalise cities. Londres: Routledge.

Tomsen, S. y Markwell, K. (2007). When the Glitter Settles: Safety and Hostility at and Around Gay and Lesbian Public Events. Newcastle (Australia): University of Newcastle.

Troitiño, M., De la Calle, M. y l, M. (2003). "Los visitantes de las ciudades históricas españolas: características generales y rasgos específicos inducidos por la celebración de eventos turístico-culturales». En: E. Ortega (coord.). Investigación y estrategia turísticas. Madrid: Thomson, 37-64.

Tubella, I. y Vilaseca, J. (coord.). (2005). Sociedad del conocimiento. Cómo cambia el mundo ante nuestros ojos. Barcelona: UOC.

Turisme de Catalunya (2013). El movimiento LGBT en Catalunya. Agència Catalana de Turisme, Barcelona. <http://catalunya-lgbt.catalunya.com/es/lgbt.php>. [Consulta: 23 febrero 2015].

Visser, G. (2002). «Gay men, tourism and urban space: reflections on Africa's gay capital». Tourism Geographies, 5 (2), 168-189. <http://dx.doi.org/10.1080/1461668032000068261>

Waitt, G. y Gorman-Murray, A. (2008). "Camp in the Country: Re-negotiating Sexuality and Gender through a Rural Lesbian and Gay Festival». Journal of Tourism and Cultural Change, 6 (3), 185-207. <http://dx.doi.org/10.1080/14766820802647616>

Waitt, G. y Markwell, K. (2006). Gay tourism. Culture and context. Nueva York: The Haworth Hospitality Press.

Waitt, G., Markwell, K. y Gorman-Murray, A. (2008). «Challenging heteronormativity in tourism studies: locating progress». Progress in Human Geography, 32 (6), 781-800. <http://dx.doi.org/10.1177/0309132508089827>

Wall, G. y Mathieson, A. (2006). Tourism: Change, impacts and opportunities. Essex: Pearson Prentice Hall.

White, N. y White, P. (2008). «Travel as interaction: encountering place and others». Journal of Hospitality and Tourism Management, 15, 42-48. <http://dx.doi.org/10.1375/jhtm.15.42>

Yüksel, A. y Yüksel, F. (2001). «Comparative Performance Analysis: Tourists’ Perceptions of Turkey Relative to Other Tourist Destinations». Journal of Vacation Marketing, 7 (4), 333-355.

<http://dx.doi.org/10.1177/135676670100700404> 